\title{
Design of stereoscopic warehouse control system based on PLC
}

\author{
Xie yongzhi, ${ }^{1, a, 2, b}$, Liang tianyu ${ }^{2, b}$ \\ ${ }^{1}$ Guangxi key laboratory of new energy and building energy saving, Guilin 541004, China \\ ${ }^{2}$ College of Mechanical and Control Engineering, Guilin University of Technology, Guilin 541004, \\ China
}

Keywords: automated warehouse; Mitsubishi PLC; stepper motor; electrical control; logistics management.

Abstract: The design of a small-scale automated warehouse for cargo storage, including mechanical structure system such as shelf, stacker and conveying rail system, and PLC controller, stepper motor and stepper motor drive are composed of high speed and high Precision electrical control system. The system has a friendly human-computer interaction, through the preparation of the PLC ladder program can achieve precise control of the stacker action. The structure of the stacker is simple, beautiful in appearance, reasonable in layout and simple in handling, and has wide practical application prospect.

\section{Introduction}

With the modern scientific and technological progress and the high degree of development of industrial production, modern logistics technology industry has produced a new warehouse storage three-dimensional warehouse. It has become more and more widely used in the field of warehouse storage around the world. It is composed of shelves, roadway stacking cranes, operation control systems and other components, is a modern logistics system in a key component, is a multi-storey storage of goods warehouse system. The new warehouse is a new type of system that does not require manual direct involvement in the automatic storage and removal of goods [1]. Through the automatic control of the conveyor system, the material is transported to the warehouse shelves. With the roadway stacking machine, to achieve the automatic delivery of materials, storage. These processes are networked managed and controlled by a computer. The research content of this paper is a key element of the automated logistics control system - the three-dimensional warehouse control system. The purpose is to optimize the efficiency of the system by optimizing the design.

\section{Mechanical structure design of stereoscopic warehouse}

The automated warehouse consists of shelves, pallets (boxes), roadway Stacking Cranes, transportation guide systems, AGV systems and operation control systems. Its basic components are:

Shelf: a structure made of steel material that holds goods [10]. The shelf is mainly composed of two basic forms: Welded shelves and combined shelves. The model is shown in figure 1:

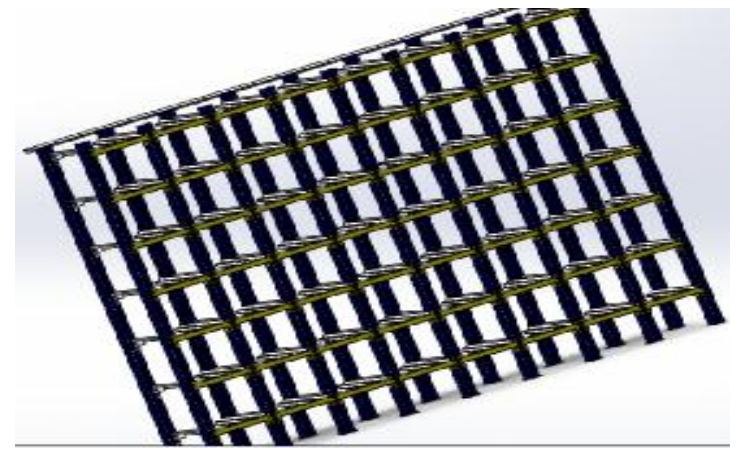

Figure 1 stereoscopic warehouse shelves

Carriage rail system: it is the peripheral equipment of the whole warehouse, mainly responsible for transporting the goods, or taking the goods away from the stacker. There are many kinds of conveyors, 
of which the most common are: roller conveyors, chain conveyors, and elevators. The model of the transport system is shown in figure 2:

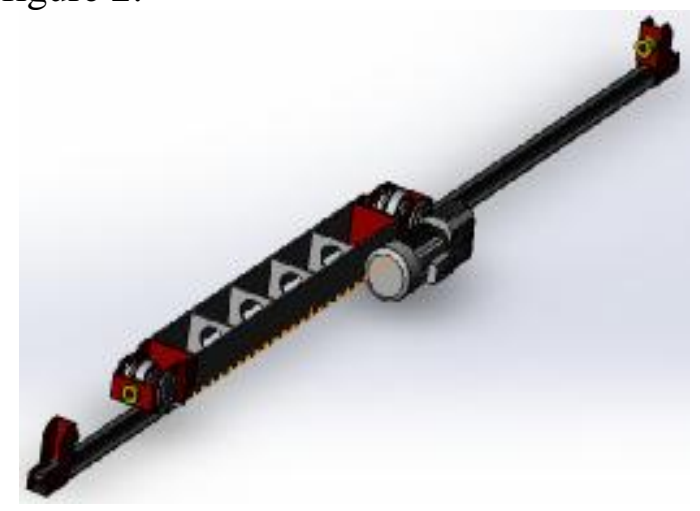

Fig. 2 conveying guide system

\section{main circuit design of electrical control system}

The schematic diagram of the main circuit of the electric control system in the automated warehouse system is shown in figure 3 .

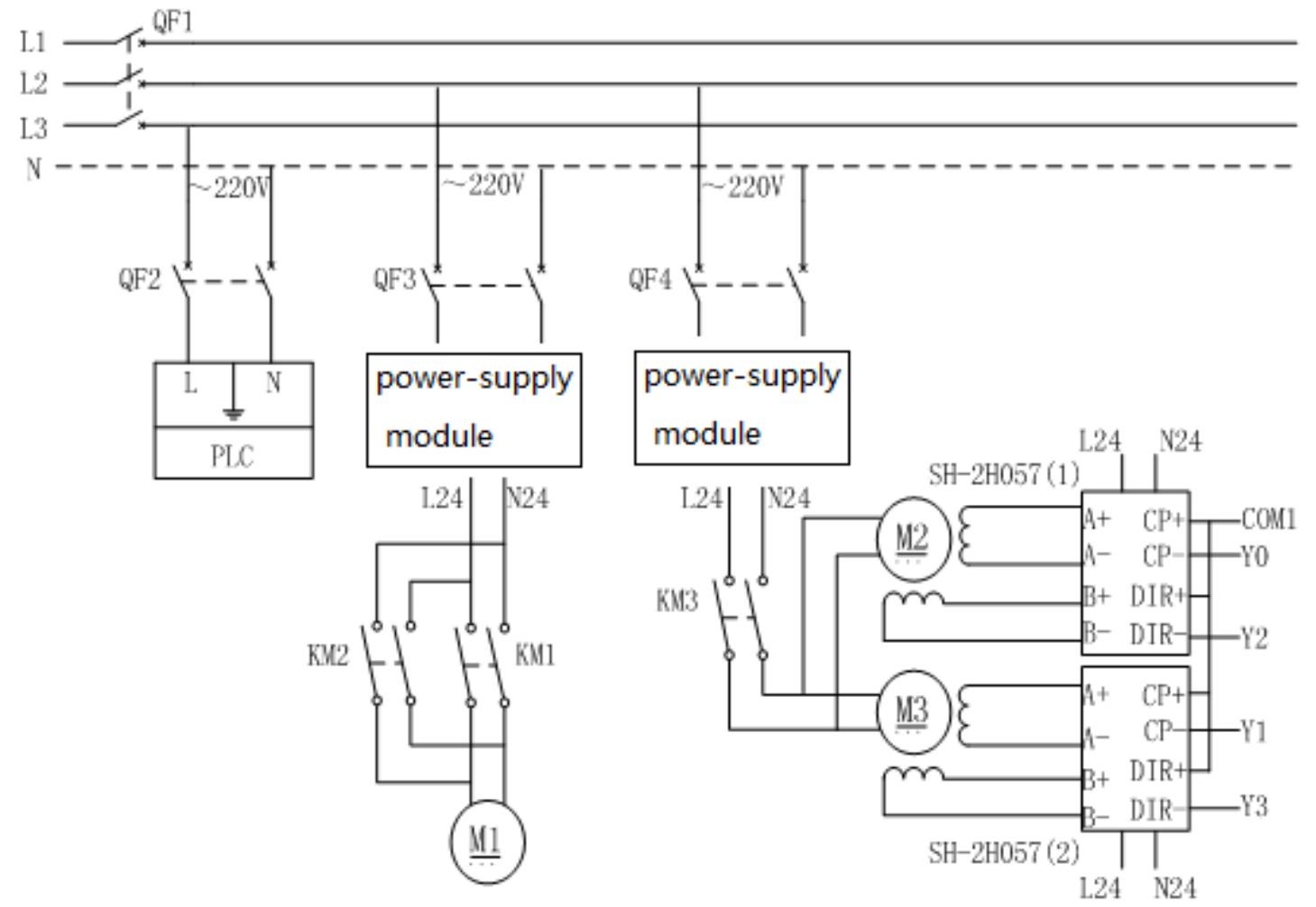

Fig. 3 Schematic diagram of main circuit

(1) In the main circuit, the $220 \mathrm{~V}$ single-phase AC is drawn out from the power grid, one part is supplied directly to the MITSUBISHI PLC, and the other part outputs the 24V DC through the power module.

(2) DC contactor KM1, KM2 control the direct and reverse rotation of Z-axis DC motor M1 to complete operation of pick and place the cargo; DC contactor KM3 control X, Y axis two stepper motor M2, M3 power supply access, and emergency shutdown in case of failure to ensure the safety of the system running. The stepper motor driver SH-2H057 (1), SH-2H057 (2) and the stepper motor M2, M3 supporting the use of, to ensure the normal drive signal input.

(3) QF1 is the main switch of power supply. It can not only protect the main circuit from short circuit, but also interrupt the three-phase AC power supply. QF2, QF3 and QF4 realize the overload and short circuit protection of each load loop, and they are easy to use and maintain. 


\section{hardware structure design of control system}

The hardware of the automatic warehouse control system is mainly composed of PLC, DC motor, stepper motor, stepper motor driver, parallel switching power supply, micro switch and Omron EE-SPY402 groove reflection type connector type sensor. The external wiring diagram for the PLC is shown in figure 4:

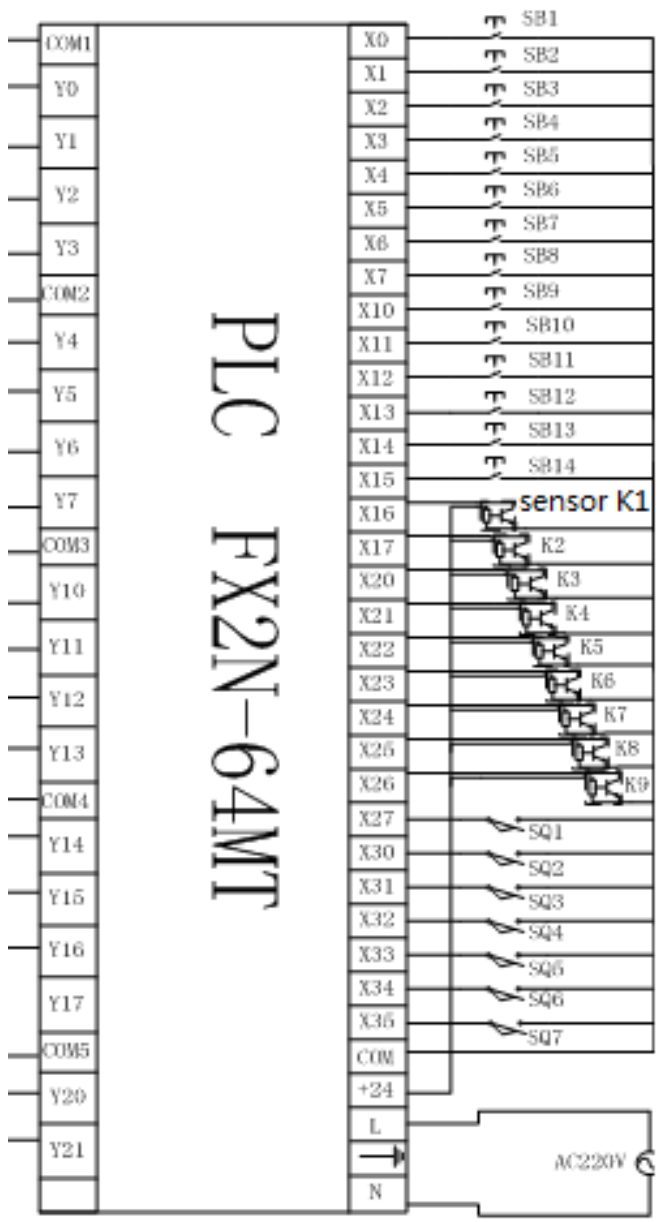

Figure 4 PLC wiring diagram

The output part: Y0 to Y21 is the output side, completing the specified run step.

(1) the two terminals of DC5V are connected with the $\mathrm{L}$ and $\mathrm{N}$ terminals of the main circuit respectively. They are used as a power supply in the PLC output circuit, respectively feeding the power output of the Y0, Y1, Y2 and Y3 in the output circuit. PLC select transistor output type, Y0 and Y1 can send 2-20kHz frequency pulse, connect stepper motor driver SH-2H057 (1), SH-2H057 (2) $\mathrm{CP}$ end, and then control stepper motor M2, M3. Y2 and Y3 connect the stepper motor driver SH-2H057 (1) and SH-2H057 (2) to the DIR end, and then control the stepping motor M2 and M3 in the direction of motion.

(2) DC24V corresponds to the main circuit of the L24, N24 terminals. The L24 is used as a power supply for the PLC output circuit, which supplies power to the Y4-Y21 connected to the output circuit respectively. In the output loop, the N24 connects to the COM2-COM5 end of the PLC. Y4 and Y5 drive the $\mathrm{Z}$ shaft DC motor respectively to perform positive and reverse movement. The coil branches of the two contactors of KM1 and KM2 are designed as a interlock circuit to prevent erroneous operation. Y21 drive relay KM3 action, in case of failure to cut the stepper motor voltage, to ensure the safety of the operation of the system. Y6, Y7 and Y20 are respectively connected with the display lamp HL1, the delivery display lamp HL2 and the operation error display lamp HL3, indicating the working state of the system at this time. Y10-Y17 connect seven sections of digital tube, Y10-Y16 connect digital tube A-G segment, in the system operation, to display the current operation of the position number. Y17 is not available because it does not require decimal point display。 
The input part: the X0-X15 terminal of PLC is connected with the input button of the system, mainly used for the input of system operation signal and position selection signal.

(1) the X16-X26 terminal of PLC connects the photoelectric sensors of 9 positions, and whether there is the detection information of the goods in the input position, it can guarantee the reliability of the system when it is running.

(2) PLC X27-X35 terminal connection 7 limit switch, which forward limit switch, reverse limit switch, the uplink limit switch for the system limit position monitoring. When the PLC receives any of the limit switch signal input, then start the display light HL3, and control the relay KM3 action, make the stepping motor to cut off the power supply itself, ensure system safety; The limit switch and the No. 0 limit switch are mainly used for self-resetting of the system. The delivery limit switch and the pick-up limit switch mainly control the action position of the fork when the goods are taken.

\section{system software design}

The system software design includes delivery subsystem, pick up subsystem, reset subsystem and fault protection subsystem. The system software design includes delivery subsystem, pick up subsystem, reset subsystem and fault protection subsystem. The system program flow as shown in Figure 5, When the delivery signal is detected at the input of the system, the delivery program begins. First of all, to detect the position of the information, to detect whether it is out of stock, if detected goods, the system display error, reset procedures, reset to the original 0 positions, the end of the operation. If no goods are detected, proceed to the next step, the horizontal X-axis stepper motor forward and position it at a horizontal position on the shelf, then the vertical Y-axis stepping motor forward and position it to a shelf Position, and then reach the designated position, Z-axis motor is running, fork into the goods. Then, the fork is lowered and the goods are placed on the shelves. Z-axis motor reversal, the fork will be recovered, the fork rose to a certain height. Perform a reset procedure, reset the stacker, etc. to the original 0 position, and end the program.

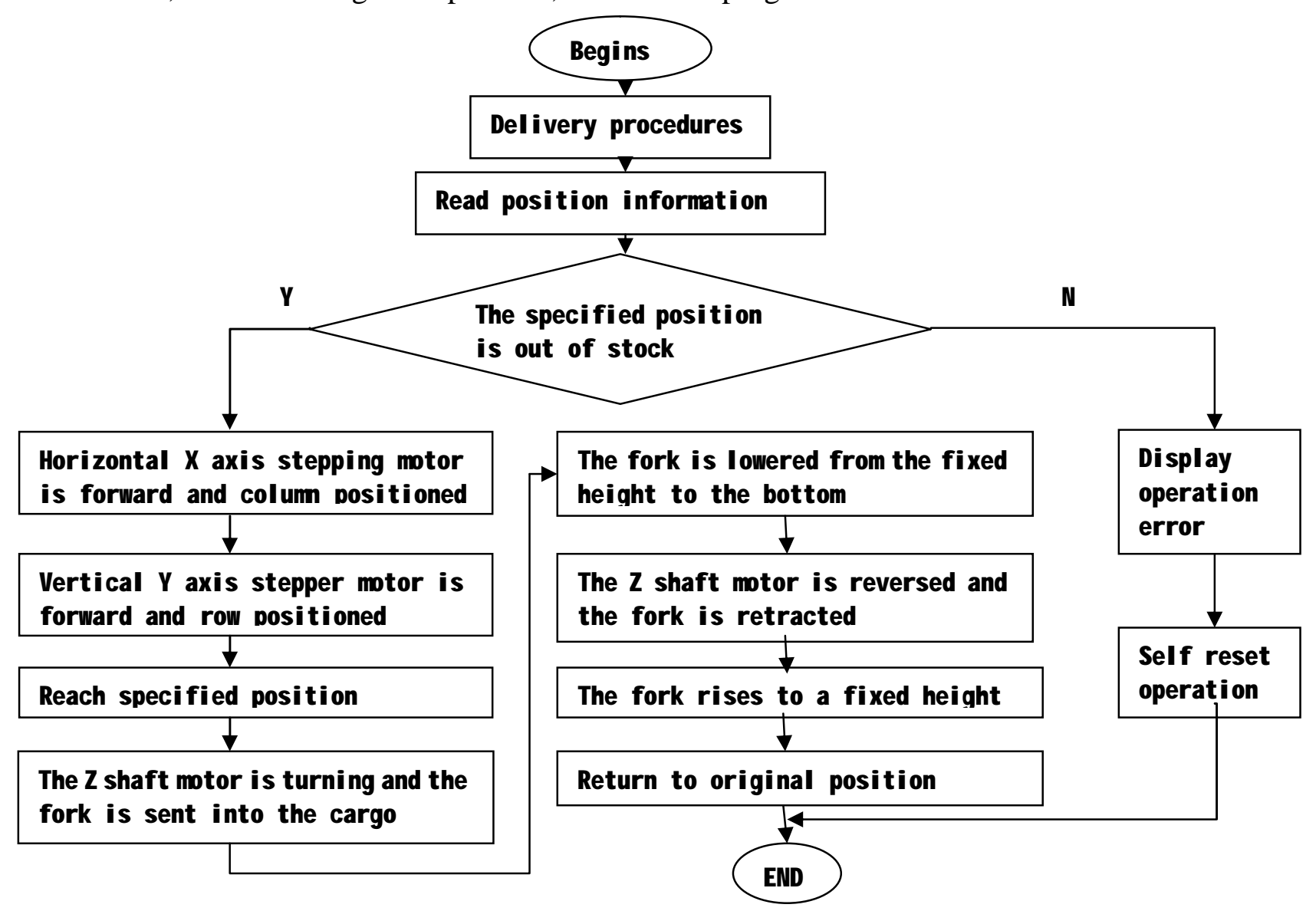

Figure 5 system delivery sub-flow chart 


\section{Conclusions}

Automated warehouse has the advantages of small ground, large storage and quick turnover. It can solve the problems of urban land shortage, high labor intensity and low efficiency. Through the design of the shelf, stacker, conveyor and the compilation of the stacker and conveyor control program, a small automatic warehouse system has been established. Its control system with MITSUBISHI PLC as the control core, has the advantages of high reliability, high speed, high sensitivity, and through the preparation of control procedures, to complete the various automated warehouse operations.

\section{Acknowledgements}

the research fund of Guangxi key laboratory of new energy and building energy saving(No. 15-J-21-12),

\section{References}

[1] Zhu Jianjun. Design of practical teaching equipment for stereoscopic warehouse[J]. Laboratory research and exploration,2017.36(1):277-286.

[2] Liu Lu, Chang Xiaoling. Design of PLC control system for stacker crane based on industrial control network[J]. Modular machine tools and automatic machining technology,2015.1:93-96.

[3] Li gang Design of teaching experiment platform for small stack crane[J]. Chinese Agricultural Mechanization,2012.240(2):165-171.

[4] Li Qingmei, Qu Xin. Design of Running System in Automatic Storage and Retrieval System Based on PLC[J]. Coal Technology,2013.32(12).

[5] Li Wei. Study on the status quo and development of original Yangcheng lake crabs cultivation[J]. Journal of Chinese Agricultural Mechanization,2014.35(5):173-186. 